\title{
Síndrome de Ogilvie em paciente portador de adenocarcinoma gástrico
}

\author{
Ogilvie's Syndrome in patients with gastric adenocarcinoma
}

Síndrome de Ogilvie en un paciente con adenocarcinoma gástrico

Lucas Brandão Damasceno Góes*, Maíra Soares Torres², Maurício Meireles Góes³.

\section{RESUMO}

Objetivo: Relatar um caso Síndrome de Ogilvie complicada e suas possíveis correlações com o adenocarcinoma gástrico. Detalhamento do caso: O presente caso é de uma paciente do sexo feminino, 69 anos, em tratamento de adenocarcinoma gástrico, que chegou ao pronto atendimento com queixa de distensão abdominal súbita, taquicardia, taquipneia, apresentando, ruídos hidroaéreos reduzidos, abdome timpânico à percussão, sem sinais de defesa ou de irritação peritoneal. $O$ estudo tomográfico revelou distensão gasosa difusa sem a presença de obstrução. A paciente evoluiu com aumento grave da pressão intra-abdominal. Foi submetida à laparotomia exploradora com achado de extensa necrose de alças intestinais e sem sinais de obstrução mecânica. Realizada íleo-colectomia total com utilização de bolsa de Bogotá. Apesar das medidas, houve piora global do quadro pós-operatório com choque refratário, levando ao óbito. Considerações finais: A carência de conhecimentos a respeito da fisiopatologia torna o manejo desses pacientes ainda mais desafiador para médicos. Além disso, o diagnóstico precoce é a chave para um melhor prognóstico.

Palavras-chave: Pseudo-Obstrução do colo, Pseudo-Obstrução colônica, Síndrome de Ogilvie, Adenocarcinoma.

\section{ABSTRACT}

Objective: To report a case of complicated Ogilvie's syndrome and its possible correlations with gastric adenocarcinoma. Details of the case: The present case is of a female patient, 69 years old, undergoing treatment for gastric adenocarcinoma, who arrived at the emergency department with a complaint of sudden abdominal distention, tachycardia, tachypnea, presenting reduced hydro-air noises, tympanic abdomen to percussion, without signs of defense or peritoneal irritation. The tomographic study revealed diffuse gas distension without the presence of obstruction. The patient evolved with a severe increase in intra-abdominal pressure. She underwent exploratory laparotomy with the finding of extensive necrosis of intestinal loops and without signs of mechanical obstruction. Total ileo-colectomy was performed using a Bogotá bag. Despite the measures, there was an overall worsening of the postoperative condition with refractory shock, leading to death. Final considerations: The lack of knowledge about pathophysiology makes the management of these patients even more challenging for doctors. In addition, early diagnosis is the key to a better prognosis.

Keywords: Colonic Pseudo-Obstruction, Ogilvie's syndrome, Colonic pseudo obstruction, Adenocarcinoma.

\section{RESUMEN}

Objetivo: reportar un caso de Síndrome de Ogilvie complicado y sus posibles correlaciones con adenocarcinoma gástrico. Detalles del caso: El presente caso es de una paciente, de 69 años de edad, sometida a tratamiento para adenocarcinoma gástrico, que llegó al servicio de urgencias con una queja de distensión abdominal repentina, taquicardia, taquipnea, presentando ruidos hidro-aéreos reducidos, abdomen timpánico a percusión, sin signos de defensa o irritación peritoneal. El estudio tomográfico reveló distensión de gas difusa sin la presencia de obstrucción. La paciente evolucionó con un aumento severo de la presión intra abdominal. Se sometió a una laparotomía exploratoria conelhallazgo de necrosis extensa de asas intestinales y sin signos de obstrucción mecánica. La ileo-colectomía total se realizó con una bolsa de Bogotá. A pesar de las medidas, hubo un empeoramiento general de la condición postoperatoria con shock refractario, que condujo a la muerte. Consideraciones finales: La falta de conocimiento sobre fisiopatología hace que

${ }^{1}$ Faculdade de Ciências Médicas de Minas Gerais (FCMMG), Belo Horizonte - MG.

*E-mail: lbdgoes@gmail.com

2Universidade Federal de Medicina (UFMG), Belo Horizonte - MG.

${ }^{3}$ Hospital da Baleia, Belo Horizonte - MG.

SUBMETIDO EM: 6/2020

ACEITO EM: 7/2020

PUBLICADO EM: 9/2020

REAS/EJCH | Vol.Sup.n.59 | e4153 | DOI: https://doi.org/10.25248/reas.e4153.2020 Página 1 de 7 
el tratamiento de estos pacientes sea aún más desafiante para los médicos. Además, el diagnóstico precoz es la clave para un mejor pronóstico.

Palabras clave: Seudo obstrucción colónica, Pseudoobstrucción colónica, Síndrome de Ogilvie, Adenocarcinoma.

\section{INTRODUÇÃO}

A Síndrome de Ogilvie, também conhecida pseudo-obstrução intestinal aguda, foi primeiramente descrita por William H. Ogilvie em 1948, com o relato de dois casos, nos quais os pacientes possuíam sintomas sugestivos de obstruções do cólon, com tumores que interromperam a inervação simpática do intestino grosso, levando a um desequilíbrio com a ação do sistema parassimpático, ocorrendo a uma dilatação importante(OGILVIE H, 1948).

É uma condição rara, com incidência de 100 casos a cada 100000 admissões (WELLS Cl, et al., 2017), representada pela dilatação aguda do colón sem a presença de fatores mecânicos obstrutivos, podendo apresentar de forma aguda ou crônica(WELLS Cl, et al., 2017;BERNARDI MP, et al.,2015).

Geralmente, acomete pacientes hospitalizados e está relacionada a várias patologias, sejam elas traumáticas, obstétricas, cirúrgicas, cardiorrespiratórias e neurológicas (MARCONDES RFJ, et al.,2009; ROSS, ET AL, 2016). A fisiopatologia e a etiologia são pouco conhecidas, porém um desequilíbrio na inervação autonômica, de origem multifatorial: inibição do reflexo motor através dos ramos aferentes esplâncnicos em resposta a um estímulo nocivo, aumento de resposta inibitória motora simpática, diminuição do estímulo excitatório e excesso de resposta inibitória sobre o parassimpático, inibição da liberação do oxido nítrico (NO) pelos neurônios, além da estimulação periférica excessiva dos receptores opioides(WELLS Cl, et al., 2017; MARCONDES RFJ, et al.,2009;SCHLITTLER LA, et al.,2010; JAIN A, VARGAS H.,2012).

O diagnóstico é realizado por exclusão, como diagnóstico diferencial de carcinomas, aderências, dilatação gástrica aguda, volvos, isquemia mesentérica, impactação fecal (WELLS Cl, et al.,2017;MARCONDES RFJ, et al.,2009) O tratamento pode ser feito de três formas: conservadora, cirúrgica ou descompressão por colonoscopia(MARCONDES RFJ, et al.,2009;JAIN A, VARGAS H.,2012).

A Síndrome de Ogilvie compromete a barreira mucosa intestinal, favorecendo a ocorrência de translocação bacteriana, que pode culminar em sepse. Pela fragilidade da barreira mucosa e da imunodeficiência associada aos quadros oncológicos, pacientes com pseudo-obstrução colônica associada a adenocarcinoma podem evoluir com choque séptico de etiologia bacteriana (normalmente Enterococcus da microbiota com padrão de virulência habitual), sendo importante causa de óbito em pacientes com esse perfil (TAHARA S, et al., 2019).

O objetivo do presente estudo é elucidar a associação do adenocarcinoma com a ocorrência da Síndrome de Ogilvie e a apresentação dos possíveis desfechos em pacientes graves para que sejam reconhecidos padrões que permitam diagnóstico mais precoce, levando a adoção de medidas que tenham impacto na redução de morbimortalidade desses pacientes. Diante da escassez de literatura sobre o tema, a confecção desse relato torna-se ainda mais relevante.

\section{DETALHAMENTO DO CASO}

Paciente feminina, 69 anos, hipertensa, diabética, dislipidêmica, tabagista (carga tabágica calculada pela fórmula maços/ano: 80), com doença do refluxo gastroesofágico e em uso de Ácido Acetilsalicílico100mg; Anlodipino $5 \mathrm{mg}$ 12/12horas, Hidroclorotiazida 25mg, Sinvastatina 20mg, Metformina $850 \mathrm{mg}$ 12/12h,Imipramina 25mg, Diazepam 10mg, Pantoprazol 40mg 12/12h, Domperidona 10mg 8/8 horas. História ginecológico obstétrica: cinco gestações, três partos, sendo duas cesárias e2 abortos. História de gastrectomia parcial com linfadenectomia das cadeias linfáticas perigástricas (linfadenectomia D1), reconstrução a Billrothll (BII) e dreno sentinela em coto duodenal em outubro de 2017, devido à adenocarcinoma gástrico.

Estadiamento: tumor primário invade peritônio visceral, apresenta metástases em 7-15 linfonodos regionais e não apresenta metástases à distância. Sendo a classificação patológica (p) das caraterísticas do tumor primário $(\mathrm{T})$, as características dos linfonodos das cadeias de drenagem linfática do órgão em que o 
tumor se localiza $(\mathrm{N})$ e a presença de metástases a distância (M): pT4apN3aM0. Em tratamento com quimioterapia e radioterapia. Paciente comparece ao Pronto Atendimento, com relato de distensão abdominal súbita, com piorano início da manhã, associado a taquipneia e taquicardia. Refere evacuações no dia anterior, com eliminação de flatos. Nega febre, vômitos e náuseas. Última sessão de quimioterapia há aproximadamente quatro dias.

Ao exame físico, paciente se encontrava afebril e anictérica, os ruídos hidroaéreos estavam reduzidos, abdome extremamente distendido, timpânico à percussão difusamente, compressível, sem sinais de defesa, sem sinais de irritação peritoneal. A radiografia de abdome evidenciou padrão de pseudo-obstrução, sem obstrução mecânica, com dilatação gasosa importante da moldura colônica. O estudo tomográfico do abdome e pelve demonstrou distensão gasosa difusa, sem fatores obstrutivos desde a transição retossigmoideana até o colón direito e leve em alças jejuno-ileais (incompetência de válvula ileocecal). Sem a presença de sinais de isquemia de alças, pneumoperitôneo, distensão do intestino delgado ou coleções endocavitárias. (Figura 1, Figura 2 A e 2B)

Devido ao quadro de dor e instabilidade clínica a paciente foi transferida para o Centro de Terapia Intensiva(CTI).O tratamento inicial foi de suporte intensivo com tentativa de controle da dor, hidratação venosa, clister glicerinado via retal, com eliminação de fezes, e Neostigmina 2,5 mg endovenoso a cada 3 horas realizado a tentativa de descompressão por sonda retal em que apresentou baixa resposta clínica com persistência da distensão abdominal, piora da dor e da mecânica ventilatória, sendo necessária intubação orotraqueal, e início da ventilação mecânica.

A tomografia computadorizada de região abdominal foi repetida após 24 horas com persistência da distensão gasosa, com alça sigmoide atingindo onze centímetros de diâmetro transverso com veia cava inferior colabada nas porções renal e suprarrenal. Foi utilizado sonda vesical de demora para mensuração da pressão intra-abdominal, que evidenciou valores de $50 \mathrm{mmHg}$, correspondendo a síndrome compartimental abdominal grau IV.

Em função dos achados, optou-se por tratamento cirúrgico. Submetida a laparotomia exploradora, foi constatado extensa necrose das alças intestinais, sendo realizado íleo-colectomia total com utilização de bolsa de Bogotá. Durante o pré-operatório houve a necessidade de drogas vasoativas (Noradrenalina). Evoluiu no CTI com quadro de choque refratário e síndrome de disfunção de múltiplos órgãos e sistemas (acidose metabólica, coagulopatia, anúria, elevação de lactato, hipotermia). Apesar das medidas instituídas a paciente evoluiu à óbito no dia seguinte ao procedimento cirúrgico (Figura 3).

Figura 1 - Tomografia de abdome em corte coronal.

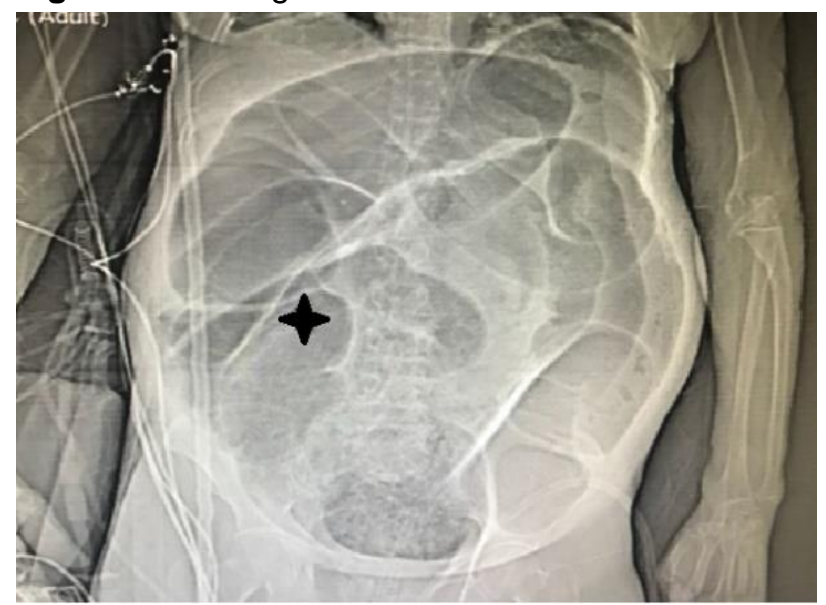

Legenda: Estudo tomográfico em plano coronal demonstrando distensão difusa, sem fatores obstrutivos, desde a transição retossigmoideana até colón direito (destaque para o asterisco preto) e leve distensão em alças jejuno ileais (incompetência da válvula ileocecal). Fonte: GÓES LBD, et al., 2020. 
Figura 2 - Tomografia de abdome em corte axial.

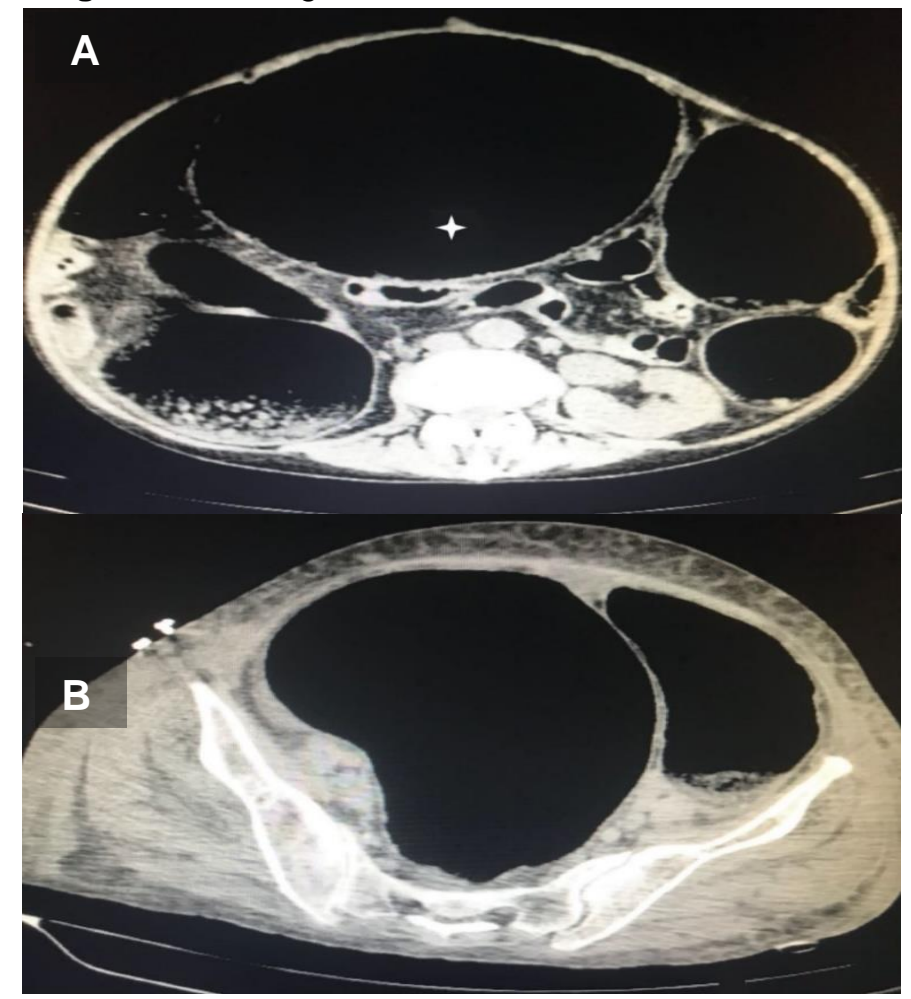

Legenda: Estudo tomográfico em plano axial referente a região abdominal (A) e pélvica (B). No abdome é observada distensão importante (com destaque para o asterisco) incluindo deslocamento das vísceras abdominais devido a dilatação das alças, é possível detectar a estase de material fecalóide de forma difusa. $\mathrm{Na}$ pelve é observado acentuada distensão gasosa difusa no reto médio, sem a presença de fatores obstrutivos, além da presença de estase difusa de material fecalóide.

Fonte: GÓES LBD, et al., 2020.

Figura 3 - Peça operatória.

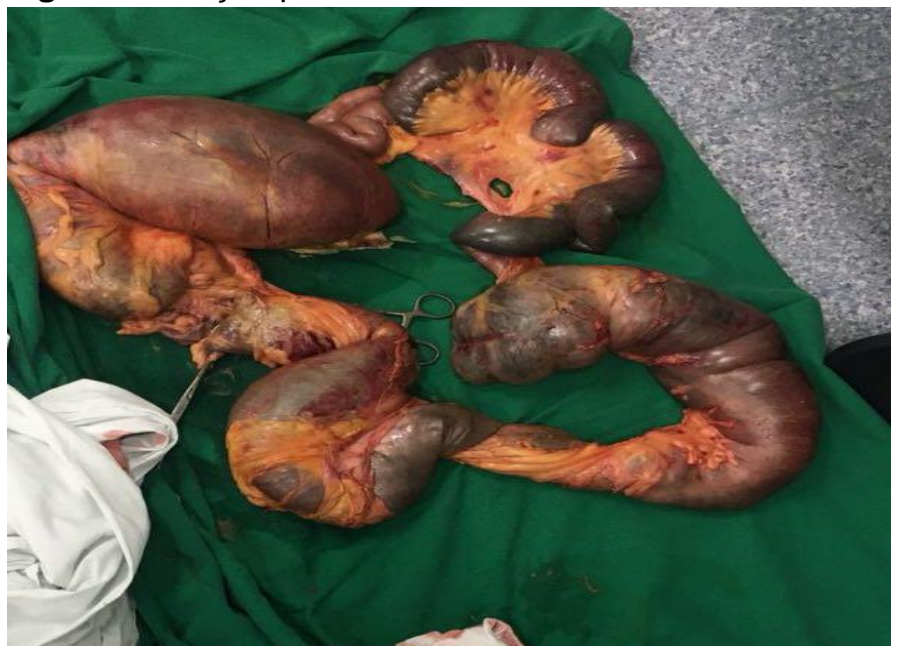

Legenda: Peça operatória, íleo-colectomia, demonstrando acentuada distensão de alças e áreas de isquemia e necrose.

Fonte: GÓES LBD, et al., 2020. 


\section{DISCUSSÃO}

A pseudo-obstrução aguda é um termo que caracteriza uma síndrome com sinais, sintomas e achados radiográficos que se assemelham a obstrução do intestino grosso, mas sem associação com fatores mecânicos (WELLS CI, et al., 2017;MARCONDES RFJ, et al.,2009; SCHLITTLER LA, et al.,2010; JAIN A, VARGAS H.,2012). A apresentação aguda pode ocorrer em qualquer idade, porém, apresenta maior incidência em pacientes idosos, 60 anos, com predominância pelo sexo masculino (60\%) (ROSS, et al., 2016).

A terapia de suporte inclui: suspensão da dieta entérica, e das medicações que afetam a motilidade cólica, como opioides, anticolinérgicos e antagonistas dos canais de cálcio (WELLS CI, et al., 2017; SCHLITTLER LA, et al., 2010).

A utilização da Neostigmina, um inibidor reversível da acetilcolinesterase, tem sido a primeira escolha na terapia medicamentosa na tentativa de regular a função autonômica cólica, caso não haja perfuração ou peritonite(SCHLITTLER LA, et al.,2010). Outros fármacos como o Tegaserode, Metoclopramida, Ibuprofeno, Indometacina, já foram testados, mas não podem ser utilizados devido à falta de evidência científica representativa(HAJ M, et al., 2018; ROSS SW, et al, 2016; PEREIRA P. et al., 2015).

A descompressão por colonoscopia é realizada caso as outras medidas terapêuticas não sejam suficientes. A intervenção cirúrgica é utilizada como última alternativa, sendo aplicada nos casos mais graves, quando existe ocorrência de isquemia, peritonite ou perfurações (GAUTAM, et al., 2020; WELLS Cl, et al., 2017; MARCONDES RFJ, et al.,2009). A laparotomia exploradora tem o potencial de auxiliar na suspeita de isquemia, dessa forma, é possível realizar a cecotomia ou a colectomia (MARCONDES RFJ, et al.,2009; SCHLITTLER L. A, et al.,2010; NADUKKANDIYIL N, et al, 2014; GAUTAM, et al., 2020).

Asíndrome de Ogilvie é um desafio para médicos devido ao pouco conhecimento existente a respeito da fisiopatologia dessa síndrome. A descrição de novos casos reforça a necessidade de conhecer os diagnósticos diferenciais e os possíveis fatores predisponentes dessa síndrome. Dessa forma, o diagnóstico precoce é a chave para a realização das intervenções que podem ser conservadoras e farmacológicas, quando a primeira não demonstra resultado. Entretanto, não existem consensos a respeito da terapêutica com Neostigmina (HAJ M, et al., 2018; ROSS SW, et al, 2016; PEREIRA P. et al., 2015)o que pode resultar na opção pelo tratamento cirúrgico que possui grande morbidade e mortalidade em até $44 \%$ dos casos(PEREIRA P. et al., 2015;BERNARDI MP, et al.,2015).

No presente caso, a existência de múltiplos fatores desencadeantes dificulta o estabelecimento de uma etiologia, dentre as quais: cirurgias prévias, patologias sistêmicas e uso de medicamentos bloqueadores de canais de cálcio e de agentes redutores da motilidade intestinal. Os quais podem promover baixa resposta a terapêutica com Neostigmina (PEREIRA P. et al., 2015). A paciente apresentou quadro de distensão abdominal importante, evidenciada na tomografia computadorizada, e ausência de ruídos hidroaéreos. Evoluiu com sinais de abdome agudo, motivo pelo qual foi realizada a laparotomia. Essa comprovou a ausência de fator mecânico para obstrução, sendo realizada uma íleo-colectomia total. A paciente faleceu por complicações da doença de base e por síndrome da resposta inflamatória aguda, que foram determinantes para o prognóstico do caso.

Muito discutida atualmente, a translocação bacteriana pode ter sido um dos fatores agravantes do quadro da paciente, somando ao estado inflamatório generalizado e comprometimento orgânico crítico. $O$ acometimento intestinal leva a aumento da permeabilidade da mucosa a bactérias comuns da microbiota intestinal de virulência habitual. Devido ao quadro inflamatório sistêmico e comprometimento imune, mesmo Enterococcus podem promover bacteremia com graves repercussões, desencadeando a liberação de mediadores e promovendo intensa agressão tecidual, deflagrando choque séptico. Como a Síndrome de Ogilvie costuma acometer pacientes debilitados, esse estado inflamatório consumptivo gera deterioração importante que muitas vezes leva a óbito. (TAHARA S, et al., 2019; HAJ M, et al., 2018).

A literatura disponível sobre Síndrome de Ogilvie é muito restrita, com poucos estudos do tipo revisão sistemática, de coorte e de caso-controle. A maior parte dos trabalhos são relatos de caso. dessa forma, faltam estudos com níveis de evidência forte que norteiem para a melhor conduta terapêutica, para 
efetivamente reduzir os desfechos negativos associados ao diagnóstico. Essa grande escassez de literatura referente à Síndrome de Ogilvie, reflete a baixa incidência, a dificuldade diagnóstica e o grande desconhecimento por parte dos profissionais de saúde que lidam com o doente, em especial o intensivista e o cirurgião.

A rápida progressão do quadro clínico torna a tomada de decisão terapêutica efetiva necessária, porém devido à necessidade de intervenção precoce, em muitos casos ocorrem percalços de comunicação entre as equipes do Departamento de Medicina Intensiva e a equipe de cirurgia, agravando a condição clínica do paciente e aumentando a morbimortalidade pela tomada de condutas intempestivas em pacientes em estado crítico. Assim, diante da suspeita de pseudo-obstrução colônica, o estabelecimento de uma comunicação efetiva e eficaz entre ambas equipes, com diálogo aberto e baseado em evidências, em busca da estabilização do paciente e posterior resolução por completo do estado inflamatório sistêmico exacerbado.

Diante de um paciente como o do caso apresentado, os autores sugerem a realização precoce de medidas de estabilização clínica, com reanimação volêmica racional, uso de drogas vasoativas e início de tratamento conservador para Síndrome de Ogilvie. A presença do adenocarcinoma gástrico aponta para fragilidade imunológica, comprometimento nutricional e disfunção do trato gastrointestinal. As condições de base devem ser avaliadas e reestabelecidas, na medida do possível, com medidas terapêuticasde resgate. Deve-se adotar abordagem multidisciplinar, englobando as equipes cirúrgicas, o serviço de nutrição, a comissão hospitalar de controle de infecções e a equipe de intensivistas.

Além, do tratamento da pseudo-obstrução do cólon, propriamente dito, deve-se atentar para o controle das repercussões da síndrome: infecções e hiperativação inflamatória. O médico assistente deve estar atento a qualquer sinal indicativo de choque séptico e síndrome de disfunção múltipla de órgãos consequente à síndrome da resposta imune grave. Diante de casos que se apresentem com graves complicações, as medidas conservadoras podem ser preteridas à descompressão cirúrgica, de acordo com literatura mais recente. (GAUTAM NM, et al., 2020).

O adenocarcinoma gástrico é quinto câncer com maior incidência mundial, apresentando altas taxas de mortalidade (JOHNSTON FM, BECKMAN M, 2019). A ressecção cirúrgica da lesão tumoral apresenta-se como a única chance de cura, porém recidivas são frequentes, mesmo com ressecção completa (JOHNSTON FM, BECKMAN M, 2019). Pode-se citar como fatores de risco a infecção gástrica por Helicobacter pylori, dietas ricas em nitritos e alimentos em conserva.

Em países ocidentais ainda não há protocolo de rastreio estabelecido em nível populacional, fato que leva a diagnóstico em estágios avançados. De acordo com a classificação de Lauren, o adenocarcinoma gástrico pode ser subdividido em intestinal ou difuso, este apresenta pior prognóstico. Pacientes com este quadro normalmente são oligo/assintomáticos, podendo apresentar perda involuntária de peso, anorexia, dispepsia, sensação de plenitude gástrica e episódios de êmese.

O diagnóstico definitivo é definido por análise histológica de biópsia de lesões suspeitas. Para avaliar a invasão local é recomendada a realização de ultrassonografia endoscópica e para a investigação de metástases a distância, a realização de tomografia computadorizada. A abordagem terapêutica deve ser multidisciplinar, com ressecção completa da lesão, com margens de 2-6 cm, associada a realização de linfadenectomia das cadeias linfáticas perigástricas (linfadenectomia D1) (JOHNSTON FM, BECKMAN M, 2019; WÖLL E, et al, 2019).

Estudos mostraram que a linfadenectomia das cadeias perigástricas e do eixo celíaco (linfadenectomia D2) apresenta menor taxa de recidiva em 5 anos, mas maiores taxas de complicações, sendo apenas recomendação em países ocidentais. Na presença de acometimento peritoneal, o tratamento cirúrgico deve ser evitado, com indicação de quimio e radioterapia (WÖLL E, et al., 2019).

O adenocarcinoma gástrico é o terceiro câncer mais letal (JOHNSTON FM, BECKMAN M, 2019). A morbimortalidade infligida ao paciente pela doença e pelo tratamento debilita 0 paciente e favorece 0 aparecimento de complicações. Dentre essas complicações, é possível citar a Síndrome de Ogilvie, que contribui para o pior desfecho associado ao adenocarcinoma gástrico. 
A compreensão dos conhecimentos teórico-práticos envolvidos na Síndrome de Ogilvie e a individualização do atendimento ao paciente, é fundamental, pois, proporciona o rápido diagnóstico e a realização de um tratamento eficiente. Dessa forma, são almejados desfechos mais favoráveis deredução da morbimortalidade em pacientes críticos e com prognóstico reservado.

\section{REFERÊNCIAS}

1. BERNARDI MP, et al. Acute and chronic pseudo-obstruction: a current update. ANZ Journal of Surgery. 2015 maio 6;85(10):709-14.

2. GAUTAM NM, et al. Colonic Decompression Reduces Proximal Acute Colonic Pseudo-obstruction and Related Symptons. Diseases of Colon and Rectum. 2020 jan; 63 (1): 60-67.

3. HAJ M, et al. Ogilvie's syndrome: management and outcomes. Medicine Baltimore. 2018jul; 97 (27): e11187.

4. JAIN A, VARGAS H. Advances and Challenges in the Management of Acute Colonic Pseudo-Obstruction (Ogilvie Syndrome). Clinics in Colon and Rectal Surgery. 2012 mar;25(1):037-45.

5. JOHNSTON FM, BECKMAN M. Updates on Management of Gastric Cancer. Current Oncology Reports. 2019 jun; 21 (8): 67.

6. MARCONDES RFJ, et al. Síndrome de Ogilvie (pseudo-obstrução intestinal aguda): relato de caso. ABCD, Arquivos Brasileiros de Cirurgiadigestiva. 2009;22(2): 124-126.

7. MEHTA R, et al. Factors predicting successful outcome following neostigmine therapy in acute colonic pseudoobstruction: A prospective study. Journal of Gastroenterology and Hepatology. 2006 fev;21(2):459-61.

8. NADUKKANDIYIL N, et al. Acute intestinal pseudo-obstruction (Ogilvie's syndrome): A case report. Journal of Clinical Gerontology and Geriatrics. 2014 dez;5(4):140-2.

9. PEREIRA P. et al. Ogilvie's syndrome-acute colonic pseudo-obstruction. Journal of Visceral Surgery. 2015 abr;152(2):99-105.

10. OGILVIE H. Large-intestine Colic due to Sympathetic Deprivation. BMJ. 1948 out 9;2(4579):671-3.

11. ROSS SW, et al. Acute Colonic Pseudo-obstruction: Defining the Epidemiology, Treatment, and Adverse Outcomes of Ogilvie's Syndrome. Am Surg. 2016; 82:102-111.

12. SCHLITTLERL. A., et al. Relato de um caso - Síndrome de Ogilvie associada a adenocarcinoma de pâncreas. J PortGastrenterol. 2010;17(2): 60-64.

13. TAHARA $S$ et al. Pneumatosis intestinalis and hepatic portal venous gas associated with gas-forming bacterial translocation due to postoperative paralytic ileus. Medicine Baltimore. 2019 jan; 98(2): e14079.

14. WELLS Cl, et al. Acute colonic pseudo-obstruction: A systematic review of aetiology and mechanisms. World Journal of Gastroenterology. 2017;23(30):5634.

15. WÖLL E, et al. Treatment Algorithm for Patients with Gastric Adenocarcinoma: an Austrian Consensus on Systemic Therapy. Anticancer research. 2019 sep; 39 (9):4589-4596. 